\title{
Loot boxes are more prevalent in United Kingdom video games than previously considered: updating Zendle et al. (2020)
}

Paid 'loot boxes' are products in computer games that consumers can purchase to obtain randomised rewards [1]. Loot boxes are structurally similar to gambling [2, 3], and loot box expenditure is correlated with problem gambling severity [4, 5]. Zendle et al. [6] influentially reported that loot boxes are prevalently implemented in United Kingdom (UK) games in February 2019: specifically, inter alia, that $59.0 \%$ of the 100 highest-grossing iPhone games contained loot boxes. Xiao et al. [7] conducted a replication and found that $77.0 \%$ of a comparable sample contained loot boxes in June 2021. Overall, $76.9 \%$ of the 52 games only appearing in Xiao contained loot boxes, whereas $63.5 \%$ did in Zendle. This suggests that games with loot boxes became more popular over the intervening time period. However, there were 11 disagreements (22.9\%) among the 48 overlapping titles, each of which involved only Xiao identifying loot boxes, whereas Zendle did not. Table 1 summarises each disagreement, and timestamped screenshots of loot boxes uniquely identified by Xiao are available at: https://doi.org/10.17605/OSF.IO/CX5RV.

Game 1 disclosed starting to sell loot boxes between the two studies [8]. Xiao also discovered relatively obscure loot boxes in four games that Zendle did not: games 2 and 3 were known to implement loot boxes during Zendle's data collection period [9, 10]; game 4 likely also did as revealed by contemporaneous evidence [11]; and game 5 potentially contains loot boxes implemented by third-parties through user-generated content. A methodological difference may have allowed for more accurate identification by Xiao: Zendle reviewed online videos recorded by other players and, if unable to decide, then through personal gameplay, whereas Xiao determined through gameplay and, if unable to decide, then through online resources. Studying video games through personal gameplay, whenever possible, is likely preferable.

Further, recovering older versions of the software to verify is now likely impossible, which is why future research studying video games should account for their easily changeable nature by following open science principles [12] (e.g. through providing screenshots).

Additionally, five games were simulated casino games [13], and a sixth game allowed players to virtually operate physical claw machines (which are an older quasi-gambling product available to children) [14]. These games bore near identical names alluding to gambling at both data collection points, so their primary content likely did not change. Zendle did not recognise certain simulated casino games in which players can spend real-world money to buy more stakes to continue participating in simulated gambling as loot boxes, although such randomised mechanics requiring payment to engage do fall within the definition of 'loot boxes' from a ludology perspective [15], as they similarly use gambling-like mechanisms, and are, therefore, relevant to policymaking concerned with addressing potential harms [16].

Paid loot boxes are now more commonly implemented in the highest-grossing UK iPhone games (which are reflective of other Western markets) than reported by Zendle. This is because of multiple reasons: games with loot boxes becoming more popular; some popular games subsequently introducing loot boxes; methodological factors around loot box identification; and semantic ambiguities around what constitutes a 'loot box.' Policymakers [17-19] and researchers should proceed on that updated basis.

\section{ACKNOWLEDGEMENTS}

Thanks to Dr. David Zendle for making the underlying data to Zendle et al. [6] publicly available for reanalysis at: https://doi.org/10.17605/ OSF.IO/XNW2T.

L.Y.X. is supported by a PhD Fellowship funded by the IT University of Copenhagen (IT-Universitetet i København), which is publicly funded by the Kingdom of Denmark.

\section{FUNDING INFORMATION}

IT University of Copenhagen (IT-Universitetet i København)

\section{DECLARATIONS OF INTEREST}

L.Y.X. was employed by LiveMe, a subsidiary of Cheetah Mobile (NYSE:CMCM) as an in-house counsel intern from July to August 2019 in Beijing, People's Republic of China. L.Y.X. was not involved with the monetisation of video games by Cheetah Mobile or its subsidiaries. P.W.S.N. is a member of the Advisory Board for Safer Gambling-an advisory group of the Gambling Commission in Great Britain, and was a special advisor to the House of Lords Select Committee Enquiry on the Social and Economic Impact of the Gambling Industry. In the last 3 years, P.W.S.N. has received research funding from Clean Up Gambling and has contributed to research projects funded by GambleAware, Gambling Research Australia, NSW Responsible Gambling Fund and the Victorian Responsible Gambling Foundation. P.W.S.N has received travel and accommodation funding from the Spanish Federation of Rehabilitated Gamblers and received open access fee grant income from Gambling Research Exchange Ontario. 


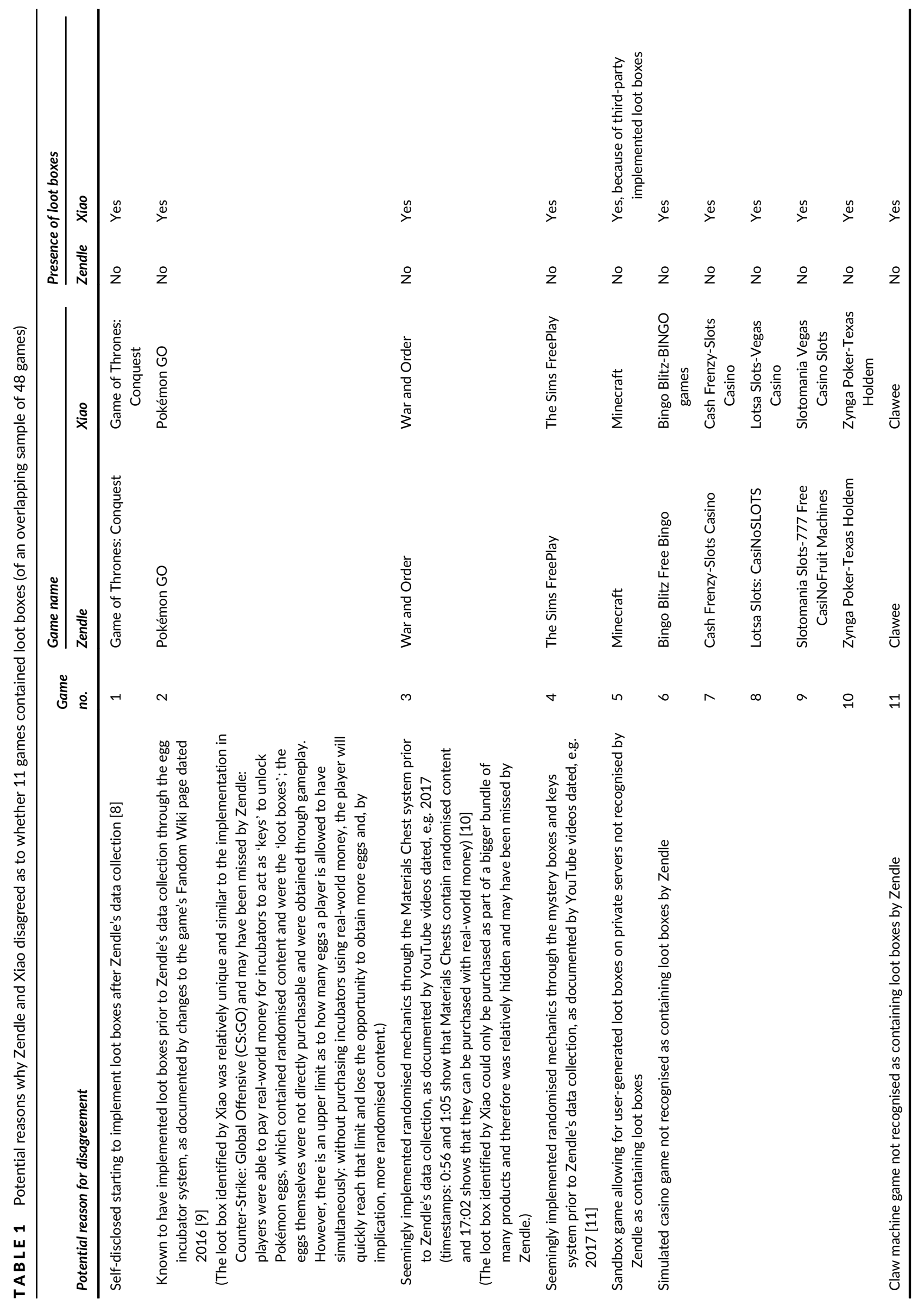




\section{AUTHOR CONTRIBUTIONS}

Leon Y. Xiao: Conceptualization; data curation; formal analysis; investigation; methodology; project administration; resources; software; visualization. Laura Henderson: Investigation; validation. Philip Newall: Conceptualization; methodology; project administration; supervision.

\section{KEYWORDS}

Consumer protection, gambling, game analysis, loot boxes, UK, video gaming

Leon Y. Xiao ${ }^{1,2,3}$ (1D

Laura L. Henderson ${ }^{3}$ (D)

Philip W. S. Newall ${ }^{4}$ (D)

${ }^{1}$ Center for Computer Games Research, IT University of Copenhagen, København, Denmark

${ }^{2}$ School of Law, Queen Mary University of London, London, UK

${ }^{3}$ The Honourable Society of Lincoln's Inn, Lincoln's Inn, London, UK

${ }^{4}$ Experimental Gambling Research Laboratory, School of Health, Medical and Applied Sciences, CQUniversity, Sydney, NSW, Australia

\section{ORCID}

Leon Y. Xiao (D) https://orcid.org/0000-0003-0709-0777

Laura L. Henderson (D) https://orcid.org/0000-0001-8098-908X

Philip W. S. Newall (D) https://orcid.org/0000-0002-1660-9254

\section{REFERENCES}

1. Xiao LY, Henderson LL, Nielsen RKL, Grabarczyk P, Newall PWS. Loot Boxes, Gambling-Like Mechanics in Video Games. In: Lee N, editorEncyclopedia of Computer Graphics and Games. Springer; 2021.

2. Drummond A, Sauer JD. Video game loot boxes are psychologically akin to gambling. Nat Hum Behav. 2018;2(8):530-2. https://doi.org/ 10.1038/s41562-018-0360-1

3. Drummond A, Sauer JD, Hall LC, Zendle D, Loudon MR. Why loot boxes could be regulated as gambling. Nat Hum Behav. 2020;4: 986-988. https://doi.org/10.1038/s41562-020-0900-3

4. Spicer SG, Nicklin LL, Uther M, Lloyd J, Lloyd H, Close J. Loot boxes, problem gambling and problem video gaming: A systematic review and meta-synthesis. New Media Soc. 2021;1-22. https://doi.org/10. $1177 / 14614448211027175$
5. Zendle D, Cairns P. Video game loot boxes are linked to problem gambling: Results of a large-scale survey. PLoS ONE. 2018;13(11): e0206767. https://doi.org/10.1371/journal.pone.0206767

6. Zendle D, Meyer R, Cairns P, Waters S, Ballou N. The prevalence of loot boxes in mobile and desktop games. Addiction. 2020;115(9): 1768-72. https://doi.org/10.1111/add.14973

7. Xiao LY, Henderson LL, Newall P. What are the odds? Lower compliance with Western loot box probability disclosure industry selfregulation than Chinese legal regulation. In OSF Preprints. 2021. https://doi.org/10.31219/osf.io/g5wd9

8. The GOT Conquest Team. Heroes Arrive in Game of Thrones: Conquest. Game of Thrones: Conquest. 2020, November 18. https:// www.gotconquest.com/news/2020/11/18/heroes-arrive-in-gameof-thrones-conquest/

9. Pokémon GO Wiki. Egg Incubators. Pokémon GO Wiki. 2020, December 15. https://pokemongo.fandom.com/wiki/Egg_Incubators

10. DaYDreaMz. WHATS THE BEST EQUIPMENT IN WAR \& ORDER. 2017, April 14. https://www.youtube.com/watch?v=Z45tKf8LatY

11. Greenoid Gemzicle. Sims Freeplay|What's in the Mystery Boxes? 2017. https://www.youtube.com/watch?v=EBHi8zIDwgY

12. Munafò MR. Opening up addiction science. Addiction. 2016;111(3): 387-8. https://doi.org/10.1111/add.13147

13. Gainsbury SM, Hing N, Delfabbro PH, King DL. A taxonomy of gambling and casino games via social media and online technologies. Int Gambl Stud. 2014;14(2):196-213. https://doi.org/10.1080/ 14459795.2014.890634

14. Newall PWS, Russell AMT, Sharman S, Walasek L. Recollected usage of legal youth gambling products: Comparisons between adult gamblers and non-gamblers in the UK and Australia. Addict Behav. 2021; 114:106685. https://doi.org/10.1016/j.addbeh.2020.106685

15. Nielsen RKL, Grabarczyk P. Are loot boxes gambling? Random reward mechanisms in video games. Trans Digit Games Res Assoc. 2019;4(3):171-207. https://doi.org/10.26503/todigra.v4i3.104

16. Xiao LY. Which implementations of loot boxes constitute gambling? A UK legal perspective on the potential harms of random reward mechanisms. Int J Ment Health Addict. 2020;1-18. Advance Online Publication. https://doi.org/10.1007/s11469-020-00372-3

17. Dirección General de Ordenación del Juego (Directorate General for the Regulation of Gambling). Proceso participativo sobre los mecanismos aleatorios de recompensa (cajas botín). 2021, February 19. https://www.ordenacionjuego.es/en/Noticia-Cajas-Botin

18. Xiao LY. Regulating loot boxes as gambling? Towards a combined legal and self-regulatory consumer protection approach. Interact Entertain Law Rev. 2021;4(1):27-47. https://doi.org/10.4337/ielr. 2021.01.02

19. Xiao LY, Henderson LL, Yang Y, Newall PWS. Gaming the system: Suboptimal compliance with loot box probability disclosure regulations in China. Behav Public Policy. Advance Online Publication. 2021;1-27. https://doi.org/10.1017/bpp.2021.23 